\title{
Rare Variant of Obturator Artery Branching from Posterior Division of Internal Iliac Artery
}

\author{
Peng Teng Chua, MBBS, MOG, FACS ${ }^{1,2}$ and Kuan-Gen Huang, MD ${ }^{3,4}$
}

\begin{abstract}
Background: An in-depth knowledge of the vascular anatomy of the female pelvis is crucial for any gyneoncologic surgeon. No two patients are the same, hence it is possible to encounter anatomical variants while performing laparoscopic gyne-oncologic surgery. In this case, we would like to report a rare variant of obturator artery origin, arising as a Y-shaped trunk from the posterior division of the internal iliac artery.

Case: A 58-year-old postmenopausal lady, with hypertension, degenerative disease of the knees, presented with abnormal uterine bleeding. Endometrial biopsy revealed atypical hyperplasia with grade 1 endometrioid carcinoma. Computed tomography scan showed an equivocal filling defect in the uterine cavity.

Result: Patient underwent laparoscopic surgical staging with total hysterectomy, bilateral salpingo-oophorectomy, with bilateral pelvic lymphadenectomy for endometrial carcinoma. Surgeons encountered an unusual branching of obturator artery from the posterior division of internal iliac artery, resembling Y shape.

Conclusion: Variants of obturator artery origin and anatomy is more common than thought, with reports in literatures ranging from $0.5 \%$ to $18 \%$ of cases. It is important to familiarize oneself with the variations as to avoid troublesome bleeding and complications during gyne-oncologic surgery. ( $\mathrm{J}$ GYNECOL SURG 36:149)
\end{abstract}

Keywords: minimal invasive gynecology, laparoscopic surgery, laparoscopic anatomy, gynecologic oncology, obturator artery, endometrial carcinoma

\section{Background}

$\mathbf{T}$ HE INTERNAL ILIAC ARTERY arises from the common iliac artery at the level of sacroiliac joint. It then passes downward, divides into anterior and posterior divisions. From the anterior division its branches are superior vesical, obturator, middle rectal, inferior vesical, inferior gluteal, and internal pudendal arteries. In females, inferior vesical artery is replaced by vaginal artery and gives an additional branch, uterine artery. The branches from the posterior division are iliolumbar, lateral sacral, and superior gluteal arteries.

\section{Case}

The patient is a 58-year-old postmenopausal lady, with hypertension and degenerative disease of the knees. She presented with abnormal uterine bleeding of 1 month duration. Endometrial biopsy revealed atypical hyperplasia with grade 1 endometrioid carcinoma. Computed tomog- raphy scan showed an equivocal filling defect in the uterine cavity.

\section{Results}

Patient underwent laparoscopic surgical staging with total hysterectomy, bilateral salpingo-oophorectomy, with bilateral pelvic lymphadenectomy for endometrial carcinoma.

During dissection of the right pelvic lymph nodes and para rectal space, we have encountered an unusual branching of obturator artery from the posterior division of internal iliac artery, with a Y-shaped trunk (Fig. 1a).

The Y-shaped trunk branched into obturator artery and another artery perforating into the right pelvic sidewall. The variant obturator artery can be traced downward toward the obturator foramen as per normal anatomical course (Fig. 1b). The variant obturator artery was carefully preserved along with its Y-shaped trunk. This variant was encountered on the right side, on patient's left side no similar variant was found.

\footnotetext{
${ }^{1}$ Consultant Obstetrician and Gynecologist, Mahkota Medical Centre, Melaka, Malaysia.

${ }^{2}$ Fellow, Gynecologic Endoscopy and Minimally Invasive Therapy, Department of Obstetrics and Gynecology, Chang Gung Memorial Hospital at Linkou, Taoyuan, Taiwan.

${ }_{3}^{3}$ Department of Obstetrics and Gynecology, Chang Gung Memorial Hospital at Linkou, Taoyuan, Taiwan.

${ }^{4}$ Department of Obstetrics and Gynecology, Chang Gung University College of Medicine, Taoyuan, Taiwan.
} 

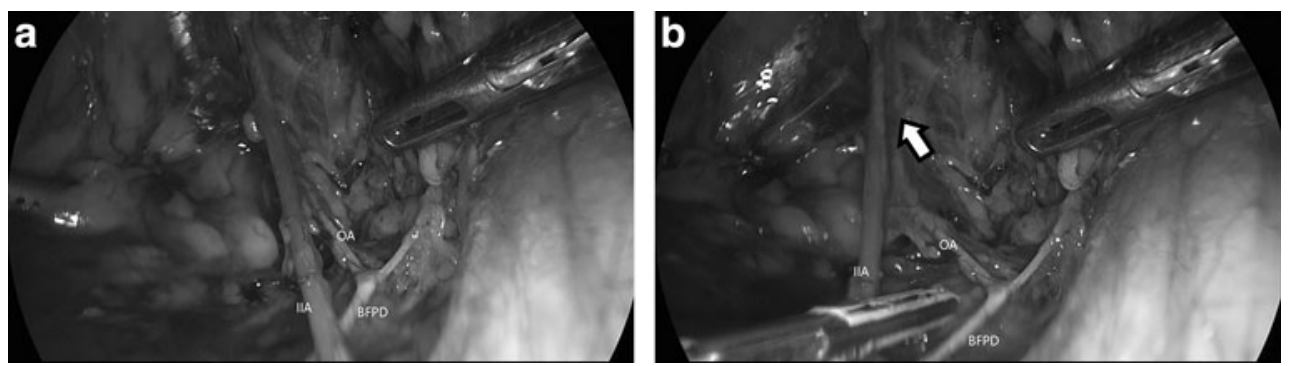

FIG. 1. Rare Y shaped obturator artery (in right pelvis) with it's vertical trunk branching from posterior division of internal iliac artery. (a) IIA, OA, and BFPD. (b) IIA, OA, and BFPD. White arrow indicates obturator artery traced downward. BFPD, branch from posterior division of internal iliac artery; IIA, internal iliac artery anterior division; OA, obturator artery.

\section{Conclusion}

Various anatomical literatures have described the variant origin of obturator artery from posterior division of internal iliac artery, ranging from $0.5 \%$ to $18 \%$ of cases. Mamatha et al. reported $14 \%^{1}$ and Pai et al. reported $3 \%$ arise as a separate branch from posterior division, whereas $4 \%$ arise with superior gluteal artery in females. ${ }^{2}$ Pavan et al. reported an incidence of $18 \% .^{3}$ Other observations include those of Pick et al. ${ }^{4} 3.28 \%$ and Kumar et al. ${ }^{5} 0.5 \%$.

It is crucial to be familiar with the variants to avoid indiscriminate injury of the said vessel during surgery, which could lead to disruption of blood supply to hip joint or neck of the femur. Vessel injury in such cases can be difficult to manage due to rapid and significant blood loss intraoperatively.

\section{Acknowledgments}

The authors thank the Asia-Pacific Association for Gynecologic Endoscopy and Minimally Invasive Therapy for providing fellowship training to P.T.C.

Institutional Review Board approval was not required for case reports based on both author's institutional regulations.

\section{Disclosure Statement}

The authors report no conflict of interest for this case report.

\section{Funding Information}

No funding was received for this case report.

\section{References}

1. Mamatha H, Hemalatha B, Vinodini P, Souza A, Suhani S. Anatomical study on the variations in the branching pattern of internal iliac artery. Indian J Surg 2012;77:248.

2. Pai MM, Krishnamurthy A, Prabhu LV, Pai MV, Kumar SA, Hadimani GA. Variability in the origin of the obturator artery. Clin Basic Res 2009;64:897.

3. Havaldar PP, Taz S, Angadi AV, Saheb SH. Morphological study of obturator artery. Int J Anat Res 2014;2:354.

4. Pick JW, Anson BJ, Ashley FL. The origin of the obturator artery. Am J Anat 1942;70:317.

5. Kumar D, Rath G. Anamolous origin of obturator artery from the internal iliac artery-A case report. Int J Morphol 2007;25:639.

Address correspondence to: Kuan-Gen Huang, MD

Department of Obstetrics and Gynecology Chang Gung Memorial Hospital at Linkou 5, Fu-Hsin Street Kweishan, Taoyuan 333 Taiwan

E-mail: kghuang@ms57.hinet.net 